\title{
Intelligent Systems for Unmanned Aircraft Safety Certification
}

\author{
Ella M. Atkins ${ }^{*}$ \\ University of Michigan, Ann Arbor, Michigan, 48109, U.S.A
}

\begin{abstract}
This paper motivates the use of intelligent decision-making algorithms onboard UAS as a means to reduce risk, particularly in anomalous or failure situations, in a manner that ultimately will enable safety certification for UAS operations throughout the National Airspace System (NAS). We begin with an overview of Federal Aviation Regulations (FARs) that must be adapted before they can be applied to Unmanned Aircraft Systems (UAS). We restrict attention to Aircraft (Subchapter C) and Airmen (Subchapter D) FARs in this paper. Specifically, we examine the regulations associated with normal and utility aircraft airworthiness (Part 23) and pilot certification (Part 61), making the assumption that UAS will initially be routinely operated in lightly-used airspace over rural areas currently occupied primarily by Part 23 aircraft operated by Part 61 airmen following Part 91 (general) operating rules. We propose a set of metrics by which both autonomous and ground-supervised risk mitigation strategies can be evaluated during the certification process to maximize the likelihood that UAS are safe when routinely operated in the NAS.
\end{abstract}

\section{Introduction}

$\mathrm{E}$ stablishing Unmanned Aircraft System (UAS) airworthiness requirements for safety certification is difficult due to regulations originally written for manned aircraft and due to the wide variation in UAS platforms, missions, and operating environments. As an interim measure to enable UAS operations prior to formidable overhead required to formally redraft and enact FARs that accommodate UAS, the Federal Aviation Administration (FAA) has interpreted the existing Federal Aviation Regulations (FARs) ${ }^{1}$ within the Code of Federal Regulations (CFRs) originally intended for manned aircraft for UAS. Current FARs classify aircraft by weight but ignore operating region and mission. Current FARs also presume the existence of "souls onboard". Certainly the UAS is distinct in that failures do not pose severe consequences (risk) unless the UAS collides with a manned aircraft or flies into terrain occupied by people or property. In fact, it is standard procedure for a UAS to terminate its flight given an anomalous event (e.g., lost link) so long as the overflight region is undeveloped and unpopulated.

Conservative application of weight-based, manned-aircraft CFRs to new UAS operations ${ }^{2}$ has stifled UAS operations in the NAS. While regulatory officials and congressional legislation may ultimately approve documents that preserve free flight for hobbyists and potentially also allow academics to fly small UAS locally without Certificates of Authorization (CoAs), these efforts are not expected to facilitate NAS access for general commercial or military users, particularly over long-range flights. UAS-specific FARs that classify by operating region as a minimum can appropriately focus attention on risk, which will certainly be acceptable for low-altitude UAS operations over unpopulated/undeveloped areas. Opening rural Class G airspace will greatly increase the ability of UAS to mature and gain trust from the community. ${ }^{3}$

Ultimately, the FARs need to support more than Class G UAS operations since many commercial and DoD UAS opportunities will require flight in urban areas, ${ }^{4}$ near busy airports, and at all altitudes. To support urban UAS operations, operating area must be augmented by other attributes for which risk can be assessed to most appropriately define airworthiness standards. Once UAS-centric FARs are approved, airworthiness certification categories should allow UAS to equitably operate ${ }^{5}$ in all airspace classes, with categories or "levels" appropriately matching certification stringency with operational risk from "no risk" (thus few certification requirements) to "high risk" (with manned-equivalent certification standards).

As we move from pilot skill and ingenuity to sophisticated avionics and data links as principle risk mitigators, we must adopt safety certification processes that are based on analytical models of the algorithms and software and are based on a concept of minimizing risk to people and property rather than to the vehicle. Given that the vehicle itself can be lost, and that small UAS in particular will not be competitive unless lightweight and low cost, electromechanical redundancy requirements must be minimized. This implies that UAS must be certifiable despite a nontrivial risk of losing the functionality of a critical component or subsystem. This, in turn, implies the UAS must be capable of adapting, in real-time, to such failures in a manner that will avoid loss-of-control in high-risk environments and that will enable onboard systems, especially under real-time pressures or with lost link, to

\footnotetext{
*Associate Professor, Aerospace Engineering Dept, University of Michigan, Ann Arbor, MI, Associate Fellow.
} 
autonomously manage the flight by computing alternate safe flight trajectories, broadcasting the nature of the disability along with updated intentions (if possible), then executing altered flight plans through safe landing or flight termination in an area where an impact poses acceptably-low risk.

Below, we first summarize safety certification requirements in existing FARs, focusing on example content in two sections, Airworthiness Standards for normal/utility airplanes (Part 23) and Certification for Pilots (Part 61). These sections were chosen because the UAS, especially the small surveillance UAS, will operate more like a general aviation "spectator" than as a scheduled or unscheduled "air carrier" transporting people or cargo. We then propose example modifications to a subset of these FARs appropriate for modern UAS equipped with full datalink, sensing, and onboard flight management capabilities. To enable UAS operation without reliance on continuous link to a ground operator/pilot even during anomalies, we propose certification requirements that rely upon, rather than accommodate as an option, intelligent systems tools that can ultimately maintain acceptable levels of risk in anomalous as well as nominal situations. Although software/system validation and verification (V\&V) are key elements of safety certification, we focus on anomaly management and procedures by which adaptive or intelligent elements can be injected without compromising V\&V of deterministic thus provable capabilities exercised during normal flight operations. We present candidate metrics by which these FARs can ultimately be deemed acceptable (or not). Such metrics are keys to ensuring certification decisions are analytic rather than ad hoc, especially given the nontrivial complexity of UAS.

\section{Summary of Relevant Federal Aviation Regulations (FARs)}

Each civil aircraft in the United States is authorized to operate in the National Airspace System (NAS) based on issuance of an Airworthiness Certificate. These certificates are issued in two varieties, either a Special Airworthiness Certificate or a Standard Airworthiness Certificate. The Standard Airworthiness Certificate issued for most massproduced aircraft is based on a production certificate, which is in turn based on a type certificate - really a design approval - issued by the FAA to the aircraft producer when the producer demonstrates that all airworthiness standards have been met or exceeded. These airworthiness standards are specified by the FARs for civil aircraft based on a division into aircraft "categories" roughly predicated on weight, means of propulsion (e.g. airplane, rotorcraft, balloon), and intended operations. These standards have evolved as a means to protect the passengers and crew onboard the aircraft, with additional considerations for other aircraft and personnel and property on the ground.

The basic aircraft categories recognized by the FAA for purposes of type certification, and subsequent issue of airworthiness certificates, are Normal Airplane, Utility Airplane, Acrobatic Airplane, Commuter Airplane, Transport Airplane, Normal Rotorcraft, Transport Rotorcraft, and Manned Free Balloons. The airworthiness standards are detailed for these categories in CFRs 23, 25, 27, 29, and 31, and the process of type certification and issuance of airworthiness certificates themselves is found in CFR 21. This presumably constitutes the bulk of aircraft certified by the FAA, but many do not fit into these categories and must have an alternative means of certification in order to fly. UAS and numerous other aircraft operate under this alternate means of authorization by way of Special Airworthiness Certificates. The FAA special airworthiness certificate authorizes aircraft to operate in the U.S. airspace and is issued in one or more of the following categories: Primary, Restricted, Limited, Light Sport, or Provisional Airworthiness Certificates, Experimental Certificates, and Special Flight Permits. These categories are specific to the Special Airworthiness Certificate, and they are delineated in a less detailed way in CFR 21 than the basic types above are defined in CFRs 23-31. In particular, CFR 21 offers little technical detail, often referring back to the CFRs describing the basic categories above.

Civil UAS currently fall into the Experimental category and are listed on the FAA web page referring to that category, but unlike various other experimental platforms they are not specifically called out in the actual wording of CFR 21. This is indicative of the uncertainties on exactly how we should regulate this new type of aircraft. It is also problematic because the regulations place a limit of one year on a certificate issued in the experimental category. While this may have been sufficient for manned flight testing it does not allow for full operational testing or deployment of UAS (for example, long duration Arctic missions), and it is extremely prohibitive in terms of economic development for companies pursuing the use of UAS for commercial purposes. This is a stark contrast to the explicit statement in CFR 21.181 (a) (4) which grants unlimited duration for amateur-built aircraft, kit-built aircraft, light-sport aircraft, air racing, and exhibition. The economic viability of UAS warrants at least the same level of acceptance once UAS are shown to introduce "acceptable" risk to people and property.

It is a challenge for the FAA to certify UAS because regulators must search through standards from multiple CFRs and other sources that were developed prior to consideration of UAS as an aircraft category. Advisory Circular 91-57 from 1981 establishes operating standards for model aircraft; this AC has now persisted for three decades. In AC 91-57, modelers are asked to operate sufficient distance from populated and noise-sensitive areas, 
avoid operation in the presence of spectators until aircraft is successfully flight-tested, avoid operation higher than 400 feet above ground level (AGL), avoid flying near full-scale [manned] aircraft, use observers if possible, and notify an airport operator if flying within three nautical miles of an airport. Because the "enjoyment" associated with model aircraft operations can be obtained through line-of-sight flight at low altitudes over unpopulated sites, numerous safety issues arising for general UAS operations, ultimately conducted in shared airspace and over populated as well as rural areas, were not necessary to address in AC 91-57. We therefore will require a more comprehensive regulatory structure, based on formal augmentations and modifications to the CFRs, to ensure new UAS operations maintain acceptable risk to people and valuable property.

Just the brief review of the CFRs above cited six different CFR parts, and most of those contain numerous pages of detailed technical specifications. A regulatory process must be developed that is specific to UAS and that will apply to the most general extent possible to both proposed and predicted platforms and use cases. Such regulations will make this process easier and more efficient, ultimately enabling UAS of all types and uses to operate routinely. Examination of existing standards will allow the UAS and regulatory communities to obtain a clear picture of which regulations can be directly applied to UAS and which cannot. UAS will likely still be categorized to an extent on weight, but flight performance characteristics, mission, and operating environment will also be important factors. UAS operations will be referenced to the existing CFRs for similar and dissimilar platforms. For example, the use of applicable "consensus standards" in the certification of light sport aircraft is reasonable to apply to UAS, both from the business use perspective and that of maintaining safety. On the other end of the spectrum, CFR 23.141 makes certification contingent on meeting standards "without requiring exceptional piloting skill, alertness, or strength," suggesting that UAS safety should also be based on averages rather than an overly-ambitious threshold applied simply because the system is unmanned.

While a comprehensive rewrite of even a single CFR section is beyond the scope of this paper, our goal is to show by example that such an analysis is possible and in fact essential to progressing toward formal certification of UAS for operation in the NAS. Example CFRs contained in Parts 23 (Aircraft) and 61 (Airmen) are therefore discussed in more detail below.

\section{Adapting Part 23: From "Manned Aircraft" to "Aircraft"}

Table 1 summarizes the subparts of CFR Part 23, Airworthiness Standards: Normal, Utility, Acrobatic, and Commuter Category Airplanes (available online at http://ecfr.gpoaccess.gov/cgi/t/text/text$\underline{\mathrm{idx}}$ ?c=ecfr\&sid=0379b6a8425e6b6e4d15e9f7595bda4a\&rgn=div5\&view=text\&node=14:1.0.1.3.10\&idno=14 ). As illustrated by Table 1, the Part 23 CFRs are extensive. A first step in parsing these CFRs for UAS is to classify them as: applicable, applicable after revision, or irrelevant. The set of "irrelevant" CFRs is nontrivial and in many cases unquestionably so (e.g., most of the Personnel and Cargo Accommodations CFRs). It may also be necessary to introduce new CFR topic areas that are critical for the certification of UAS but not for manned aircraft, most obviously inclusion of the datalink and ground station as critical and necessary augmentations to the traditional "aircraft" which previously consisted strictly of the flight hardware and (onboard) cockpit to support the flight crew.

Consider the Controllability and Maneuverability CFRs (23.143-23.157) as an example CFR series that must be adapted for UAS, and in fact for fly-by-wire for Part 23 aircraft. CFR 23.143 (General) states: "(a) The airplane must be safely controllable and maneuverable during all flight phases including-(1) Takeoff; (2) Climb; (3) Level flight; (4) Descent; (5) Go-around; and (6) Landing (power on and power off) with the wing flaps extended and retracted. (b) It must be possible to make a smooth transition from one flight condition to another (including turns and slips) without danger of exceeding the limit load factor, under any probable operating condition (including, for multiengine airplanes, those conditions normally encountered in the sudden failure of any engine). (c) If marginal conditions exist with regard to required pilot strength, the control forces necessary must be determined by quantitative tests. In no case may the control forces under the conditions specified in paragraphs $(a)$ and $(b)$ of this section exceed those prescribed in the following table. [This table, omitted here for brevity, specifies values in pounds force applied to the relevant controls (stick, wheel, rudder pedal) for temporary and prolonged application.]" Parts (a) and (b) of this CFR are general thus applicable to platforms for which a software-based autopilot is responsible for issuing control commands. Part (c) is not applicable to UAS, and is also not really applicable to fly-by-wire systems, as it prescribes pilot "strength requirements" appropriate for aircraft with mechanical linkages between pilot stick/wheel/rudder pedals and the aircraft control surfaces. ${ }^{\dagger}$ At face value, this CFR suggests a simple translation of this part (c) CFR to servo/motor actuation torque requirements, the specific

\footnotetext{
${ }^{\dagger}$ FAR 25.143, the analogous regulation for transport aircraft, includes verbage to limit applied control force to aircraft with "conventional wheel type controls". FAR 25.143 also contains text to constrain stick force limits to not being "so low that the airplane can easily be overstressed inadvertently", addressing fly-by-wire for today's transport but not unmanned aircraft where the operator will supervise rather than "fly-by-link".
} 
numerical values for which would be a function of the maximum torques each servo/motor must apply in marginal or worst-case (e.g., high-speed) flight conditions. However, embedded in this CFR is the implicit assumption that a pilot (or autopilot) must always maintain authority to move control surfaces. A pilot-centric translation of the "control force requirement" statement might be "the pilot (or remote operator) must be able to manipulate the control surfaces under all nominal and marginal operational conditions". A more general translation applicable across pilot/autopilot modes is "control surface manipulation must be possible across all nominal and marginal operational conditions". This implies that the pilot/autopilot system must be able to compute and reliably execute commands. This requires software and fly-by-wire (or mechanical) hardware to function properly under all conditions. If a ground-based pilot is safety-critical for UAS control under marginal conditions, this CFR also requires adequate pilot controls on the ground and a reliable bidirectional aircraft - ground communication link.

Table 1: CFR Part 23 Contents. ${ }^{1}$

\begin{tabular}{|c|c|c|}
\hline Subpart & Topic & CFR Sections \\
\hline A (General) & - & $23.1-23.3$ \\
\hline \multirow[t]{8}{*}{ B (Flight) } & General & $23.21-23.33$ \\
\hline & Performance & $23.45-23.77$ \\
\hline & Flight Characteristics & 23.141 \\
\hline & Controllability and Maneuverability & 23.143-23.157 \\
\hline & Trim & 23.161 \\
\hline & Stability, Stalls \& Spinning & $23.171-23.221$ \\
\hline & Ground \& Water Handling Characteristics & $23.231-23.239$ \\
\hline & Miscellaneous Flight Requirements & $23.251-23.253$ \\
\hline \multirow[t]{7}{*}{$\mathrm{C}$ (Structure) } & General & $23.301-23.307$ \\
\hline & Flight Loads; Control Surface and System Loads & $23.321-23.415$ \\
\hline & Horiz. Stabilizing, Balancing, Vertical Surfaces & $23.421-23.445$ \\
\hline & Ailerons and Special Devices & $23.455-23.459$ \\
\hline & Ground Loads / Water Loads & $23.471-23.537$ \\
\hline & Emergency Landing Conditions & $23.561-23.562$ \\
\hline & Fatigue Evaluation & $23.571-23.575$ \\
\hline \multirow[t]{9}{*}{$\mathrm{D}$ (Design\&Const.) } & General & $23.601-34.629$ \\
\hline & Wings & 23.641 \\
\hline & Control Surfaces \& Control Systems & $23.651-23.703$ \\
\hline & Landing Gear; Floats and Hulls & $23.721-23.757$ \\
\hline & Personnel and Cargo Accommodations & $23.771-23.831$ \\
\hline & Pressurization & $23.841-23.843$ \\
\hline & Fire Protection & $23.851-23.865$ \\
\hline & Electrical Bonding \& Lightning Protection & 23.867 \\
\hline & Miscellaneous (Leveling means) & 23.871 \\
\hline \multirow[t]{6}{*}{ E (Powerplant) } & General & $23.901-23.943$ \\
\hline & Fuel System / Fuel System Components & $23.951-23.1001$ \\
\hline & Oil System, Cooling/Liquid Cooling & $23.1011-23.1063$ \\
\hline & Induction System & $23.1091-23.1111$ \\
\hline & Exhaust System & $23.1121-23.1125$ \\
\hline & Powerplant Controls/Accessories/Fire Prot. & $23.1141-23.1203$ \\
\hline \multirow[t]{4}{*}{$\mathrm{F}$ (Equipment) } & General & $23.1301-23.1309$ \\
\hline & Instruments: Installation & $23.1311-23.1337$ \\
\hline & Electrical Systems and Equipment & $23.1351-23.1367$ \\
\hline & Lights, Safety, Miscellaneous Equipment & $23.1381-23.1461$ \\
\hline \multirow[t]{3}{*}{ G (Oper. Limit./Info.) } & General & $23.1401-23.1529$ \\
\hline & Markings and Placards & $23.1541-23.1567$ \\
\hline & Airplane Flight Manual; Approved Material & $23.1581-23.1589$ \\
\hline
\end{tabular}

CFR 23.145, on longitudinal control, states (subtopic headers are in bold): "(a) With the airplane as nearly as possible in trim at $1.3 V_{S 1}$, it must be possible, at speeds below the trim speed, to pitch the nose downward so that the rate of increase in airspeed allows prompt acceleration to the trim speed with-(1) Maximum continuous power on each engine; (2) Power off; and (3) Wing flap and landing gear- (i) retracted, and (ii) extended. (b) Unless otherwise required, it must be possible to carry out the following maneuvers without requiring the application of single-handed control forces exceeding those specified in $\$ 23.143(c) \ldots$ [series of specific maneuvers is omitted for brevity]. (c) At speeds above $V_{M O} M_{M O}$, and up to the maximum speed shown under $\$ 23.251$, a maneuvering 
capability of $1.5 \mathrm{~g}$ must be demonstrated to provide a margin to recover from upset or inadvertent speed increase. (d) It must be possible, with a pilot control force of not more than 10 pounds, to maintain a speed of not more than $V_{R E F}$ during a power-off glide with landing gear and wing flaps extended, for any weight of the airplane, up to and including the maximum weight. (e) By using normal flight and power controls, except as otherwise noted in paragraphs (e)(1) and (e)(2) of this section, it must be possible to establish a zero rate of descent at an attitude suitable for a controlled landing without exceeding the operational and structural limitations of the airplane... [specific conditions (e)(1) and (e)(2)omitted for brevity]." CFR 23.145 (a) is largely applicable to UAS, given that a fixed-wing UAS may be simpler, operating with no flaps or landing gear. CFR 23.145 (b) must be translated to the UAS in a manner similar to the translation of 23.143(c), with a requirement for the autopilot and/or ground-based pilot to be capable of carrying out a nominal list of longitudinal control maneuvers required throughout a flight. CFR 23.145(c) is directly translatable, although most UAS will be capable of greater than $1.5 \mathrm{~g}$ maneuvers. CFR 23.145(d) and (e) again need verbage to require the autopilot and/or ground-based pilot to be capable of maintaining power-off glide and zero-rate-of-descent attitudes suitable for controlled landings. Note that the companion CFR for transport (25.145) is analogous but with focus on the use of high-lift devices.

As examples of Part 23 CFRs that require more substantial rewrite, consider the Part 23 CFRs for Emergency Landing Conditions, $\$ 23.561$ (general) and $\$ 23.562$ (dynamic conditions). These CFRs stipulate requirements (e.g., g loads) for which aircraft must be designed to protect occupants. These CFRs are extensive, with acceleration/deceleration limits specified for each direction relative to the occupant, with specifics on motion of components including restraints and seats during the dynamic landing event, and contact forces including a head injury criteria (HIC). UAS have no occupants, thus it would be logical to classify these CFRs as irrelevant for UAS. Certainly 23.561 and 23.562 are irrelevant for UAS as written. Instead, UAS occupants and structures in the emergency landing environment on the ground must be considered. CFRs appropriately (and realistically) regulating UAS emergency landing characteristics, likely varying by vehicle class (micro, small, large), would cut to the core of the safety certification requirement to "protect people and property", thereby addressing the major safetyrelated concerns about UAS. Rather than rewrite 23.561 and 23.562 for UAS, we propose the addition of new UASspecific CFRs, e.g., 23.563 and 23.564, to describe constraints for UAS operations during emergency landing. Note that the determination of specific values for "acceptable risk" is beyond the scope of this research, and it is not clear such numbers should be hard-coded into the CFRs.

(a) The airplane, although it may be damaged or destroyed in emergency landing conditions, must be designed as prescribed in this section to protect people and property under those conditions.

(b) If the aircraft is operating in an area known to pose no or acceptable risk to people/property on the ground, the aircraft must execute an immediate emergency landing or flight termination sequence to minimize risk to other aircraft or of transiting outside the safe operating area.

(c) If the aircraft can reach a low-risk landing region, and total risk associated with transit to the low-risk site and landing is lower than risk associated with an immediate landing, the aircraft must be maneuvered to that site where it must execute an emergency landing or flight termination procedure. ${ }^{*}$

(d) If the aircraft must execute an emergency landing in an environment that may pose risk to people or property, these risks must be acceptable, or else the UAS must not operate in or near such environments. Procedures for executing such an emergency landing must be established through analysis and testing analogous to that described in CFRs 23.561 and 23.562 for manned aircraft. Manufacturers must demonstrate that: (i) Under the most common emergency landing scenarios (e.g., unmanageable winds or loss-of-thrust but intact airframe) an acceptably low impact energy is imparted to objects in the environment, (ii) Impact sites, even in a high-risk environment, will absorb the impact energy safely or will minimize risk.

\section{Figure 1: Alternative Part 23 CFR for UAS Emergency Landing.}

Consider the small UAS 'general' emergency landing regulation, e.g., 23.563, proposed in Figure 1. While such a regulation could appear oppressive at first glance, it can instead provide quantifiable means to certify UAS as acceptably safe even if there is nontrivial probability (e.g., greater than $10^{-9}$ per flight hour) of an emergency over or near a populated or improved area. Such assurance is a valuable alternative to triple redundancy as a means for certification, particularly for micro to small UAS that must remain low-cost to be competitive.

\footnotetext{
$¥$ Specifics associated with estimating total risk are to be determined. A ground-based UAS operator, given time, would upload a judgment call on local landing vs. transit to the UAS; an autopilot would estimate relatively risks quantitatively.
} 
In this section, we have shown examples of Part 23 CFRs that require adaptation (e.g., 23.143) and replacement (e.g., 23.561) for applicability to UAS. The identified differences, which cut across other CFRs more generally, are in migration from a piloted vehicle to an automatically-piloted vehicle, and in migration of safety constraints from protection of onboard occupants to protection of people and property in the environment.

\section{Adapting Part 61: From "Airmen" to "Remote Supervisors"}

CFR Part 61 specifies certification requirements for pilots, flight instructors, and ground (classroom) instructors. Subparts include: A (General), B (Aircraft Ratings and Pilot Authorizations), C (Student Pilots), D (Recreational Pilots), E (Private Pilots), F (Commercial Pilots), G (Airline Transport Pilots), H (Flight Instructors...), I (Ground Instructors), J (Sport Pilots), and K (Flight Instructors with a Sport Pilot Rating). The Sport Pilot categories, the newest Airmen classifications, were added in 2004 as an alternative that imposed fewer requirements than the standard Private Pilot rating. The differences between supervising the UAS remotely versus residing onboard the aircraft being flown are substantial. We therefore posit a new CFR subpart (potentially Subpart L?) will be most appropriate for UAS, and in fact it may be the case that multiple subparts are needed to distinguish the requirements for different classes of UAS operations (e.g., small vs. large platform, local versus long-range, low versus highaltitude, surveillance versus transport).

We are concerned with the "Airmen" who operate UAS. Unlike Part 23, the Part 61 regulations are centered on the notion that the pilot is immersed in an environment from which the aircraft is operated. Even in $\$ 61.1$, applicability and definitions, the use of the phrase "in an aircraft" is commonplace, implying the pilot must physically reside in the aircraft to obtain, for example, aeronautical experience or cross-country time. Perhaps one of the more controversial CFRs the FAA has adapted for UAS CoA operators is paragraph $\S 61.23$ regarding medical certificates. Certainly there is no distinction between manned and unmanned aircraft operation in this CFR, but aside from commenting that a ground-based UAS operator is not exposed to a thin atmosphere or unusual g-forces, it is beyond the scope of this paper to analyze an appropriate level of medical certification (if any) required for civil UAS operators.

The role of the UAS "Airman" is still evolving, but it is generally viewed to be more supervisory than hands-on piloting. ${ }^{2}$ For this reason, knowledge-based testing requirements adapted from the General Part 61 regulations will likely be more relevant for UAS than will the existing practical testing CFRs. To-date, the FAA appears to have adapted Subpart F (Commercial Pilot) regulations when possible to UAS operations, presuming the primary civil UAS user will have a commercial or military application rather than be operating "privately" or as a "sport pilot". CFRs 61.121 through 61.133 currently apply to commercial pilots. Applicability, eligibility and aeronautical knowledge requirements are mostly transferrable to UAS Airmen, except for 61.125 content regarding use of simple instruments such as a magnetic compass for manual pilotage. Aeronautical knowledge in 61.125 is insufficient for UAS. The operator must understand the available UAS instrumentation, communication protocols, operational modes and protocols. The operator must understand the set of commands and the logic behind issuing each, and the operator must understand the meaning of data transmitted by the UAS. The tradeoff in training is that the UAS supervisor need not be as proficient with "stick-and-rudder" flight control ( $\$ 61.127$ - Flight proficiency) as would a traditional general or commercial aviation pilot. The operator must know how the UAS autopilot executes maneuvers, and must know when and why the UAS would not be able to execute a maneuver, but the UAS pilot need not be able to manually execute a pinpoint landing, for example. The other Part 61 Subpart F regulations, focusing on privileges and hours of experience, including night flying, require practical adaptation to the UAS operator but are reasonable models of information to be included in UAS operator CFRs.

\section{Evaluation Metrics for Safety Certification}

The manned aircraft CFRs have evolved based on our long heritage of manned aviation. While the remotelypiloted aircraft community has existed for over half a century, this community has not been so careful to capture their experiences in a manner that facilitates translation to safety certification. The UAS community is rapidly evolving, and safety certification standards must be sufficiently accommodating to support this evolution. For this reason, it is important to develop evaluation metrics for safety certification that generalize to the extent possible. Our long-term goal, shared by others in the UAS community, is to develop a set of joint UAS \{type-missionenvironment\} attributes that translate to risk-based classification metrics, providing guidance in appropriate classification and definition of UAS-specific CFRs. Identified metrics will be critical for developing and distinguishing a minimal but flexible set of UAS-related CFRs that support the spectrum of proposed and potential future surveillance, transport, recreation, etc. mission scenarios, environments from rural to urban, and platform scales and types from micro to large transport, from quadrotor to flapping to traditional fixed-wing. Table 2 
summarizes a candidate metric set we propose for consideration, with some data acquired from the Cambone et al UAS Roadmap. ${ }^{2}$ Figure 2 shows a cross-section of small UAS applicable for a wide variety of local-area operations, while Figure 3 shows two of the most common long-range, high-altitude large UAS models operated by public and civil US agencies, Predator and Global Hawk. Figure 4 shows an example UAS ground control station developed by NASA; while this example is intentionally similar to a transport aircraft cockpit, the UAS pilots remain necessarily separated from the UAS, a critical distinction that will impact numerous CFRs as illustrated above for 23.143. As is clear from these figures, the set of possible UAS configurations is perhaps even more varied than the set of manned aircraft configurations for which the CFRs were defined. Their operational uses are equally diverse. While UAS CFRs must be responsive to the formidable set of possible attribute-value combinations depicted in Table 2, the number of distinct classifications must be minimized to avoid attempts to develop an unmanageable set of UAS CFRs.

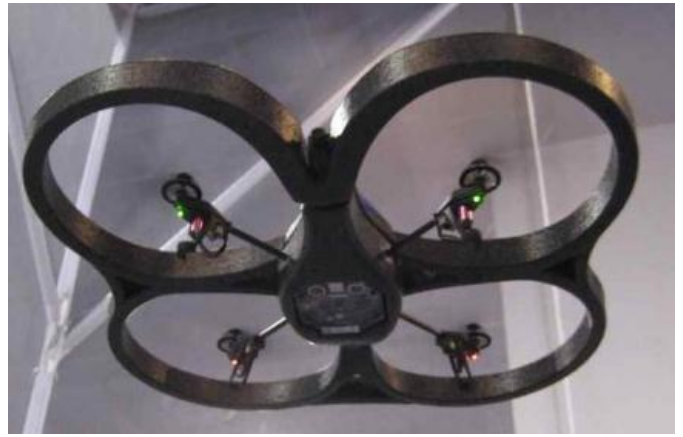

Indoor/Outdoor platforms (e.g., Quadrotor)

(Parrot AR.Drone, http://ardrone.parrot.com/parrot-ar-drone/usa/)

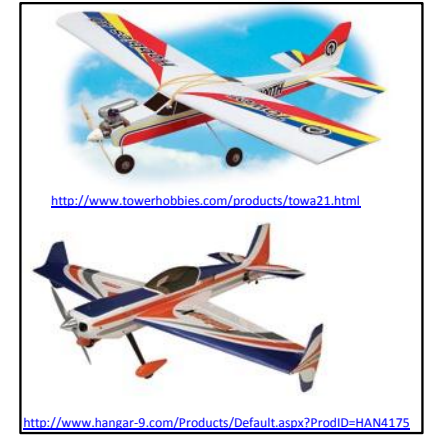

Aerobatic R/C “Conversions"

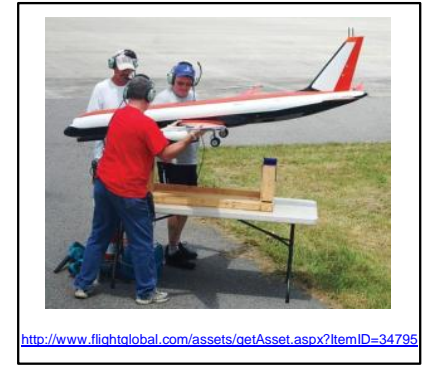

Manned Aircraft Simulation via UAS (NASA's AirSTAR)

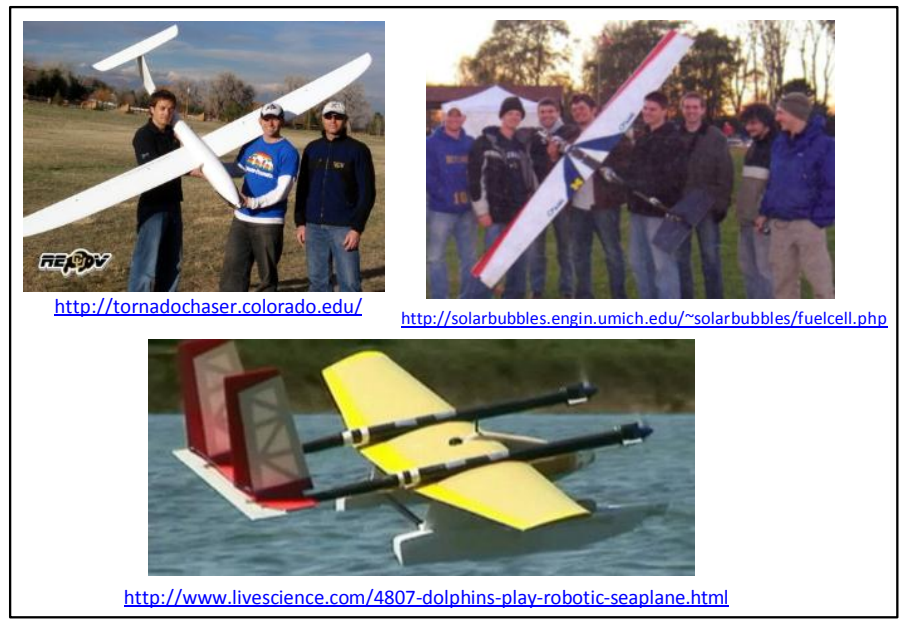

University Aeronautics \& Science Research Platforms

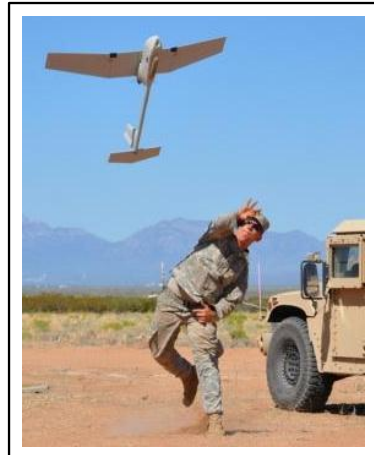

Raven (http://www.avinc.com/) Photo:

http://www.nationaldefensemagazine.org/ blog/Lists/Posts/Post.aspx?ID=388

Commercial SUAS Platforms

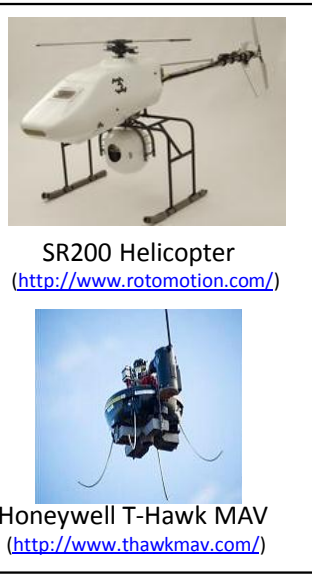

Honeywell T-Hawk MAV
(http://www.thawkmav.com/)

Figure 2: Example Small UAS Platforms.

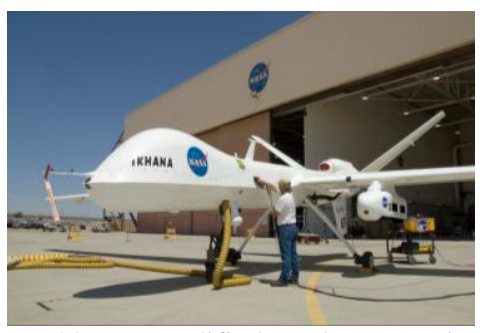

a) Ikhana (modified Predator B) Aircraft.

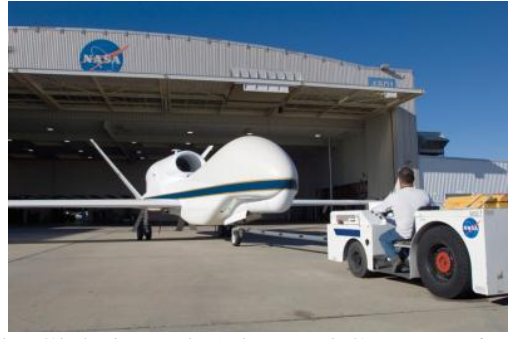

b) Global Hawk Advanced Concept Aircraft.

Figure 3: Example Large UAS Platforms ; aircraft shown here are operated by NASA Dryden Flight Research Center, http://www.nasa.gov/centers/dryden/aircraft/index.html. 


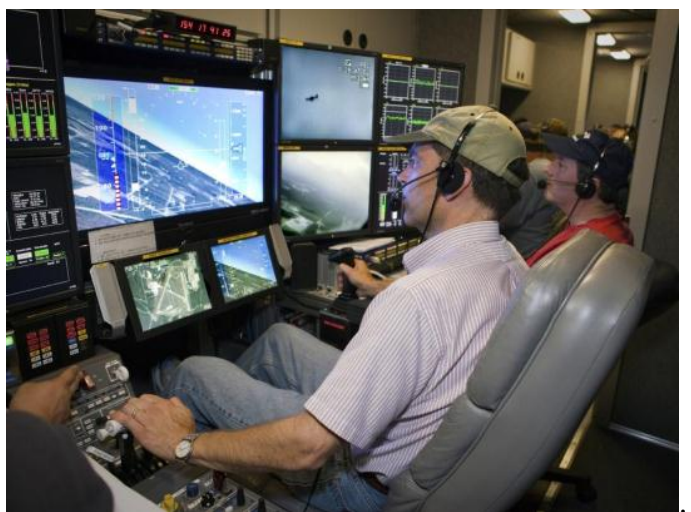

Figure 4: Example UAS Ground Station (NASA's AirSTAR) ${ }^{\S}$

http://www.nasa.gov/centers/langley/multimedia/iotw-airstar.html

Table 2: Potential UAS Classification Metrics.

\begin{tabular}{|c|l|}
\hline Attribute & \multicolumn{1}{|c|}{ Value } \\
\hline Airframe Type & Fixed-wing, rotary-wing, flapping-wing, glider, lighter-than-air, hybrid \\
\hline Weight/Mass & Group 1 (<20 lbs), Group 2 (21-55 lbs), Group 3 (<1320 lbs), Group 4/5 (>1320 lbs) \\
\hline Propulsion/Power & Single/multi-engine, propeller/jet/other, fuel/electric/solar/other \\
\hline Avionics & $\begin{array}{l}\text { Communication link(s), flight management system (autopilot) capabilities, onboard sensors, } \\
\text { sense-and-avoid equipment, ground station / operator interface }\end{array}$ \\
\hline Trim States & $\begin{array}{l}\text { Maximum endurance/range/maneuverability trim states for climb, cruise, descent, loiter } \\
\text { (i.e., airspeeds, flight path angles turn radii) }\end{array}$ \\
\hline $\begin{array}{c}\text { Flight Envelope } \\
\text { Constraints }\end{array}$ & Minimum/maximum airspeed, flight path angle, turn radius, flight ceiling, range, endurance \\
\hline $\begin{array}{c}\text { Mission } \\
\text { Airspace Class }\end{array}$ & $\begin{array}{l}\text { Recon/surveillance, attack, communications, science, entertainment, transport, agriculture } \\
\text { priority w/r/t other airspace users) }\end{array}$ \\
\hline $\begin{array}{c}\text { Class A, B, C, D, E, G, (indoor) } \\
\text { Typensic }\end{array}$ & None, low, moderate, high $\},\{$ manned/unmanned $\}$ \\
\hline Area of Operation & Rural/suburban/industrial/urban, structure/paved/turf/sand/forest/crop/water, flat/rugged \\
\hline
\end{tabular}

Airworthiness certification must fundamentally be based on safety. UAS operations have a critical difference not envisioned by the current CFRs: they can crash and not pose risk to people or valuable property. For UAS, safety is only compromised when potentially damaging kinetic energy is deposited by the UAS onto a manned aircraft or into terrain potentially occupied by people or property. Energy is a function of aircraft mass and relative speed on impact, while risk of impact with occupied terrain or other aircraft is a function of airspace class, area of operation, traffic density, and robustness of avionics/automation as well as the ground station link. UAS type informs safety certification regulations in terms of the range of energies the platform might deposit, the manner in which the UAS (and its operator) can minimize or redirect UAS energy to minimize or eliminate potential risk, and the set of critical systems that must be functioning to ensure the UAS can manage and direct its energy in a manner that maintains acceptable risk levels. UAS missions define the spectrum of flight profiles/plans over which safety must be preserved. These will inform the analyst regarding expected airspace occupation, predictability/availability of 4D flight plans, and the suite of maneuvers the UAS might elect over its mission (e.g., 4D waypoint following, target following, loitering, aggressive maneuvers). The UAS environment is defined in terms of expected and actual traffic density/types/missions as well as overflight region attributes. Environments will range from rural with no other air traffic expected to urban with high air traffic densities. The CFRs must ultimately cover the spectrum of \{type, mission, environment\} classes, building from the simplest \{small UAS, low-altitude maneuvers, UAS test

\footnotetext{
$\S$ UAS ground stations typically contain glass cockpit and video elements shown here. While no regulatory standards exist for UAS ground station design, the NASA AirSTAR configuration appears more like a traditional cockpit than do most UAS ground stations due to the team's desire to emulate the transport aircraft cockpit to the extent possible.
} 
range)\} case for which current flight termination procedures are acceptable given that UAS energy deposited on impact will be transferred only to unimproved terrain. The above attribute sets must be translated to quantitative metrics (e.g., for energy, environment) over which CFR categories may be established and evaluated. With this paper we encourage the community to establish metrics appropriate across the different types, missions, and environments each operator is targeting. Once such metrics are established, we can formally analyze the alternatives for remote decision-making versus autonomous (intelligent systems) algorithms over the spectrum of situations we anticipate as well as those scenarios we don't expect until they are encountered and catalogued, further refining the UAS-centric or UAS-accommodating CFRs as the community further matures.

\section{Conclusions and Future Work}

This paper has examined the Federal Aviation Regulations (FARs) for aircraft (Part 23) and airmen (Part 61) with the goal of identifying their applicability for UAS operations. While most of the CFRs examined require some adaptation, many of these changes are minor, with major changes revolving around the migration from onboard pilotage to onboard autonomous flight management with remote supervision and from minimizing risk to aircraft occupants to minimizing risk to people and property on the ground and in other [manned] aircraft. We proposed adaptation/extension of Part 23 to establish UAS-relevant airworthiness standards, because many existing regulations could be readily adapted. Alternatively, a new subpart of the CFRs could be created, addressing gaps in automation, instrumentation, and the link to an offboard ground station as a critical part of the system. Such specifics are beyond the scope of this paper but are critical to consider in future work. Addition of one or more new Part 61 subparts for UAS operators will also be required, consistent with the numerous subparts already in place for airmen ranging from sport to airline transport pilots. We proposed the development of safety evaluation metrics that incorporate attributes that are derived from UAS type, mission, and operating environment, since risks to people and property imposed by the UAS is necessarily a function of all these attributes. This paper introduces far more challenges than it addresses. A full revision of the CFRs to accommodate UAS is a long-term process that must be supported by the UAS community, the FAA, and ultimately by the public. Careful evaluation of safety for emerging UAS and their missions in the context of the CFRs as well as software and system risk analysis represents an essential step toward integration in the NAS.

\section{References}

${ }^{1}$ Federal Aviation Administration, FAR/AIM 2011: Federal Aviation Regulations / Aeronautical Information Manual, Aviation Supplies \& Academics, Inc., 2011.

2 Cambone, S. A., Krieg, K., Pace, P., and Linton, W., Unmanned Aircraft Systems Roadmap 2005-2030, Office of the Secretary of Defense, Aug. 2005.

3 Atkins, E., "Certifiable Autonomous Flight Management for Unmanned Aircraft Systems," The Bridge, Vol. 40, No. 4, pp. 35-43, National Academy of Engineering, Winter 2010.

4 E. Atkins, A. Khalsa, and M. Groden, "Commercial Low-Altitude UAS Operations in Population Centers," ATIO Conference, AIAA, Hilton Head, SC, Sep. 2009.

5 E. Atkins, "Metrics for Equitable UAS Operations in Mixed-Use Airspace," Unmanned Systems North America, AUVSI, Denver, CO, Aug. 2010.

${ }^{6}$ E. M. Atkins, I. Alonso-Portillo, and M. J. Strube, "Emergency Flight Planning applied to Total Loss of Thrust," Journal of Aircraft, AIAA, 43(4):1205-1216, Jul-Aug 2006. 